\title{
PROOF OF A CONJECTURE OF FRIEDMAN
}

\author{
DONALD A. MARTIN
}

\begin{abstract}
We prove that every uncountable hyperarithmetic set has a member of each hyperdegree $\geqslant 0$, the hyperdegree of Kleene's 0 .
\end{abstract}

We improve the main result of Friedman [1] by proving his conjecture that every uncountable hyperarithmetic set has a member of each hyperdegree $\geqslant 0$, the hyperdegree of Kleene's 0 . This result has been obtained independently by Friedman by a different method. Friedman's proof uses ideas employed by L. Harrington to obtain a partial result.

In [2] it is shown that there is a function $f: \omega \rightarrow \omega$ of hyperdegree 0 and a Gödel number $e$ such that $(\forall k)(f(k) \leqslant g(k))$ implies that $f$ is hyperarithmetic in $g$ with Gödel number $e$.

By [1] it suffices to prove that each recursive tree of finite sequences of natural numbers with uncountably many branches has a branch of each hyperdegree $\geqslant 0$. Let $T$ be such a tree and let $f$ be as above. Let $x: \omega \rightarrow 2$ have hyperdegree $\geqslant \mathbf{0}$. Let $A$ be the Cantor-Bendixson perfect subtree of $T$. $A \in \Sigma_{1}^{1}$. A point in $A$ is good if it has at least two immediate successors in $A$.

We shall define a branch $h$ through $A$. Let $\sigma_{0}<\sigma_{1}<\cdots$ be the good points on our branch. $\ln (\sigma)$ is the length of the finite sequence $\sigma . h\left(\ln \left(\sigma_{n}\right)\right)$ will always be given either the smallest or the next to smallest possible value. $h\left(\ln \left(\sigma_{2 n}\right)\right)$ and $h\left(\ln \left(\sigma_{2 n+1}\right)\right)$ will have the minimal possible value unless there are exactly $k$ numbers $m<n$ such that

$$
h\left(\ln \left(\sigma_{2 m}\right)\right) \text { is not minimal, and } f(k) \leqslant \ln \left(\sigma_{2 n}\right) \text {. }
$$

When this happens, $h\left(\ln \left(\sigma_{2 n}\right)\right)$ will not be minimal and $h\left(\ln \left(\sigma_{2 n+1}\right)\right)$ will be minimal just in case $x(k)=0$.

For $g: \omega \rightarrow \omega$ let $g \in C$ if and only if, for each $k$, there are at least $2 k+1$ good points $\sigma_{n}$ of length $\leqslant g(k)$ such that $h\left(\ln \left(\sigma_{n}\right)\right)$ is not minimal. $C$ is $\Sigma_{1}^{1}$ in $h$ and $g \in C \Rightarrow(\forall k)(g(k) \geqslant f(k)) . f$ is hyperarithmetic in $h$ since

$$
f^{*}=f \Leftrightarrow(\exists g \in C)\left(f^{*} \text { is hyperarithmetic in } g \text { with Gödel number } e\right) \text {. }
$$

Since both $f$ and $A$ are hyperarithmetic in each of $x$ and $h, x$ and $h$ are hyperarithmetic in one another.

\section{REFERENCES}

1. H. Friedman, Borel sets and hyperdegrees, J. Symbolic Logic 38 (1973), 405-409.

2. C. Jockusch and R. Soare, Encodability of Kleene's 0, J. Symbolic Logic 38 (1973), 437-440.

The Logic Group, Rockerfeller University, New York, New York 10021

Received by the editors January 6, 1975.

AMS (MOS) subject classifications (1970). Primary 02F35. 